\title{
História do campo da Alimentação e Nutrição em Saúde Coletiva no Brasil
}

\author{
History of the scientific field of Food and Nutrition \\ in Public Health in Brazil
}

Francisco deAssis Guedes de Vasconcelos ${ }^{1}$

M alaquias Batista Filho ${ }^{2}$

${ }^{1}$ Departamento deN utrição, Centro deCiências da Saúde, UniversidadeFederal deSantaCatarina. Campus Universitário, Trindade. 88970-600 Florianópolis SC. fguedes@floripa.com.br ${ }^{2}$ Instituto de M edicina Integral Prof. Fernando Figueira, Recife.
Abstract This paper aims to trace the history of the scientific field of Food and Nutrition in Public $\mathrm{H}$ ealth in Brazil (FNPH), trying to describe the contexts that allowed the construction, consolidation and redefinition of the field, to identify the Brazilian scientists who contributed to this process and address the scientific concepts or paradigms that guided the actions of thesescientists. As methodological criteria of analysis three cross sections corresponding to contexts of history in this field were established: the first one covers the period from 1930 to 1963, thesecond onebetween 1964 and 1984 and the third one considers the period from 1985 to 2010. We conclude that the field of FNPH assumed the current configuration from the mid-1970's, within the so-called Brazilian health movement, whose main ideas were the achievement of health reform and the construction of the Unified Health System. We reaffirm theimportance of multidisciplinary character and the premise of the important role of the field of $\mathrm{FNPH}$ in ensuring the human right to healthy food, a component of the set of conditions necessary for health promotion, disease prevention, surveillance and health recovery and improvement of quality of life for all Brazilians.

Key words Food and Nutrition, Public Health, Scientific field, Paradigms, Brazilian scientists, History of Nutrition
Resumo 0 objetivo do artigo é traçar a história do campo da Alimentação e N utrição em Saúde Coletiva (ANSC) no Brasil, procurando descre ver os contextos que possibilitaram sua construção, consolidação e ressignificação; identificar os cientistas brasileiros que contribuíram com este processo e abordar os paradigmas que nortearam a atuação destes cientistas. Como critérios metodológicos de análise foram estabelecidos três cortes transversais, correspondentes a contextos da história deste campo: o primeiro referente ao período 1930 a 1963; o segundo entre 1964 a 1984 e o terceiro considera o período 1985 a 2010. De preende-se que o campo da ANSC assumiu a atual configuração a partir de meados de 1970, no interior do chamado movimento sanitário brasileiro, cujo ideário principal foi a realização da reforma sanitária ea construção do Sistema Ú nico de Saúde. Reafirma-se a importância do caráter multidisciplinar bem como a premissa do relevante papel do campo da AN SC na garantia do direito humano à al imentação saudável, um componente do conjunto de condições necessárias à promoção da saúde, prevenção de doenças, vigilância erecuperação da saúde emelhoria da qualidade de vida de todos os brasileiros.

Palavras-chave Alimentação e Nutrição, Saúde Coletiva, Campo científico, Paradigmas, Cientistas brasileiros, História da Nutrição 
Introdução

O objetivo deste artigo étraçar a história do campo da Alimentação e N utrição em Saúde Coletiva (ANSC) no Brasil, procurando descrever os distintos contextos que possibilitaram a sua gê nese, consolidação e ressignificação; identificar os cientistas brasileiros que contribuíram para este processo e abordar as concepções científicas ou paradigmas que nortearam a atuação desses cientistas.

Como critérios metodológicos de análise e estruturação do artigo foram estabelecidos, de forma intencional, três cortes transversais, correspondentes a contextos específicos da história deste campo do conhecimento científico no Brasil: o primeiro corresponde ao período 1930 a 1963, momento em que se analisa o processo de gênese do campo; o segundo se situa no período de 1964 a 1984, identificado como o momento de consolidação do campo; finalmente, o terceiro corresponde ao período 1985 a 2010, em que se aborda a incorporação de novos paradigmas e a ressignificação do campo.

Antes de tudo, cabe explicitar que o conceito decampo científico utilizado neste artigo seguea orientação teórica da Sociologia Reflexiva, dePierre Bourdieu (1930-2002) 1,2; em simultâneo, os conceitos de paradigma e comunidade científica são oriundos da Filosofia Idealista, de Thomas Samuel Kuhn (1922-1996) 3 .

Para Kuhn ${ }^{3}$, paradigmas são as realizações científicas universalmente reconhecidas que, durante al gum tempo, fornecem problemas e soluções modelares para uma comunidade de praticantes de uma ciência. Ou ainda, conforme esclarece 0 próprio autor: um paradigma é aquilo que os membros de uma comunidade partilham e, inversamente, uma comunidade científica consiste em homens que partilham um paradigma.

$\mathrm{Na}$ concepção idealista de Kuhn ${ }^{3}$, a comunidade científica seria uma estrutura autônoma e autorreprodutora, em que os cientistas cooperariam de forma neutra e desinteressada em nome do progresso científico. De acordo com $\mathrm{Kuhn}^{3}$, para compreender o conhecimento científico como propriedade intrínseca de uma determinada comunidade científica, torna-senecessário conhecer as características essenciais dessas unidades que 0 criam e 0 utilizam. Em linhas gerais, para Kuhn ${ }^{3}$, a organização e o funcionamento da comunidadecientífica ocorriam da seguinte forma: U ma comunidade científica é formada pelos praticantes de uma especialidade científica. Estes foram submetidos a uma iniciação pro- fissional ea uma educação similares, numa extensão sem paralelos na mai oria das outras disciplinas. N este processo absorveram a mesma literatura técnica e dela retiraram muitas das mesmas lições. N ormalmente as frontei ras dessa literaturapadrão marcam os limites de um objeto de estudo científico e em geral cada comunidade possui um objeto de estudo próprio.

Rompendo com a noção kuhniana de comunidade científica, adotamos a concepção de campo científico proposta por Bourdieu ${ }^{1}$, que o concebe como um espaço de luta pelo monopólio da competência científica que é socialmente reconhecida a um agente determinado, ou seja, a capacidade técnica e o poder social de falar e intervir legitimamente em matéria de ciência. Neste sentido, para Bourdieu ${ }^{1,2}$, o campo científico constitui um mercado particular caracterizado pela luta concorrencial travada entre cientistas e/ou instituições científicas em torno da posse do capital científico - uma espécie de capital simbólico, não monetário, e que se transforma em autoridade ou competência científica para os praticantes da ciência.

Ressalta-se ainda que compartilhamos da ideia de que, na atualidade, o campo da Alimentação eN utrição estaria vivencian do uma era pósgenômica, caracterizando-secada vez mais como uma ciência ou campo científico multidisciplinar, constituído pela integração de dimensões biológicas, sociais e ambientais ${ }^{4-6}$. Dentro deste campo mais amplo está contido o campo específico da ANSC, o qual será objeto de nossa investigação neste artigo.

\section{A gênese do campo (1930 a 1963)}

É consenso na literatura científica afirmar que o campo da Alimentação eN utrição, dentro detrês grandes dimensões (ciência, profissão e política pública do Estado brasileiro), teve sua emergência no final da década de 1930, no decorrer do primeiro Governo Vargas ${ }^{7-10}$.

Como ciência, a origem do campo encontraseassociada à disciplina "H igieneAlimentar", área de estudo constituída a partir de meados do sé culo XIX nas faculdades de medicina e que, na década de 1930, possibilitaria a institucionalização acadêmica deste novo campo científico. A partir da década de 1930, duas principais vertentes de conhecimento confluíram para a conformação desse campo científico. A primeira, vertente biológica, congregava cientistas preocupados com aspectos clínico-fisiológicos eindividu- 
ais relacionados ao consumo e à utilização biológica dos nutrientes. A segunda, vertente social, reunia aqueles cuja atenção voltava-se para os aspectos econômico-sociais e populacionais relacionados à produção, à distribuição e ao consumo de alimentos. N esta segunda vertente, encontra-se a matriz para a constituição do campo da ANSC 7,9,10.

Como profissão, a partir de 1939 identifica-se a criação dos primeiros cursos para formação de profissionais em Alimentação e Nutrição (nutrólogos, nutricionistas/dietistas, auxiliares de alimentação, economistas domésticas etc.). A partir deentão, começa a ser estabel ecida a demarcação das fronteiras definidoras dos limites de competência, deautonomia e de poder entre as distintas especialidades profissionais conformadoras do complexo e interdisciplinar campo da Alimentação e Nutrição que se constituía no país $5^{9,10}$.

Como política pública, nas distintas conjunturas compreendidas no período 1930-1963, entreo Estado Novo deGetúlio Vargas (1937-1945), - Plano SALTE (saúde, alimentação, transporte eenergia) deGaspar Dutra (1946-1950), o retorno do populismo de Getúlio Vargas (1951-1954), o desenvolvimento rápido do Plano de M etas de Juscelino Kubitschek (1955-1960) e as reformas debase de João Goulart (1961-março de 1964), 0 campo materializou-se no Brasil a partir da instituição da "ração essencial mínima" (DecretoLei $n^{0} 399$, de 30 de abril de 1938) e do salário mínimo (Decreto-Lei ${ }^{\circ} 2.162$, de $1^{\circ}$ de maio de 1940); da criação do Serviço de Alimentação da Previdência Social - SAPS (D ecreto-Lei n ${ }^{\circ} 2.478$, de 5 de agosto de 1940) e da Comissão $N$ acional deAlimentação - CN A (Decreto-Lei no 7.328, de 17 de fevereiro de 1945); da instituição da Campanha $\mathrm{N}$ acional de M erenda Escolar (D ecreto ${ }^{\circ}$ 37.106, de 31 demarço de 1955), embrião do atual Programa Nacional de Alimentação Escolar (PNAE), entre outras intervenções estatais. Ou seja, no período em apreço, a intervenção estatal em Alimentação e Nutrição materializou-se a partir de ações e programas de assistência alimentar e nutricional voltados aos trabal hadores vinculados à previdência social; às gestantes, nutrizes e crianças menores de cinco anos deidadee aos escolares. Ressalta-se a íntima articulação desses programas brasileiros de assistência alimentar e nutricional com as organizações internacionais e os programas internacionais de ajuda alimentar, criados no pós-Segunda Guerra Mundial, tais como a FAO (Food and Agriculture Organization), o Unicef (United Nations International Children Emergency Fund), o Pro- grama deAlimentos paraa Paz daUSAID (Agency for International Development, dos Estados Unidos da América) e o Programa Mundial de Alimentos (PMA) $7,11,12$.

Em relação aos cientistas protagonistas do processo de construção do campo no Brasil, em estudos anteriores identificamos que 0 espaço geográfico deatuação destes cientistas deu-seinicialmente em instituições públicas localizadas, inicialmente, no eixo Rio de Janeiro-São Paulo, principais centros urbano-industriais do país, para em seguida descentralizar-se para outros estados, entre os quais se destacam Pernambuco, Bahia e Alagoas, 10,13,14. Na perspectiva social, no Rio de Janeiro, destaca-se a atuação de Josué de Castro e seus pares do Instituto de Nutrição da Universidade do Brasil, entre eles Hélio Vecchio Alves M aurício, Pedro Borges, Walter Silva (CNA) e Walter Santos (CNA); destaca-se também a atuação de Dante $N$ ascimento Costa na condução do SAPS e dos cursos para formação de profissionais do setor. Em São Paulo, Francisco Pompêo do Amaral e Geraldo Horácio de Paula Souza. Em Pernambuco, Nelson Chaves, Gilberto Freyre, Ruy Coutinho, Jamesson Ferreira Lima e Orlando Parahim. Na Bahia, Thales de Azevedo. Em Alagoas, Nabuco Lopes ${ }^{7,9,10,15}$.

Ressalta-se que, embora o perfil do cientista do campo da Alimentação e Nutrição tenha uma característica hegemonicamentemasculina, também se observou a presença feminina neste recorte inicial. N este sentido, destacam-se as atuações da química Emília Pechnik (INUB), da enfermeira/dietista LieselotteO rnellas (enfermagem da UB), das médicas Irma Fioravanti (INUB) e $N$ aíde Teodósio (PE), das nutricionistas/dietistas N eide Gaudenci, D ebble Smaira Pasotti, I one Cintra de Souza, Dalva Oliani, Eunice deO Oliveira e Arcelina Ribeiro (Superintendência do Ensino Profissional do Estado de São Paulo) e Enilda

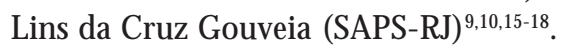

Em relação às concepções científicas ou paradigmas que nortearam a atuação dos cientistas do campo no período 1930-1963, pode-se afirmar que em um primeiro momento, circunscrito às décadas de 1930-1940, a matriz teórica que unificou os intelectuais do campo foi a tese "do valor eugênico da alimentação na construção da nação edo homem brasileiro" ou "do mal defome e não de raça". M atriz ideológica que, na época, procurava desfocar da questão racial para a questão sociocultural o preconceito racial/climático quesetinha sobreo povo brasileiro, notadamente sobre o processo de mestiçagem no Brasil. N este sentido, os cientistas do campo afirmavam a tese 
do aprimoramento eugênico do povo brasileiro mediante uma alimentação racional. No segundo momento, circunscrito ao período 1950-1963, pode-se dizer que a matriz teórico-cognitiva sobregênese, reprodução eenfrentamento da fome/ subnutrição da população brasileira defendida pelos intelectuais da Alimentação e N utrição encontrava-se em sintonia com as teorias do nacional desenvolvimentismo, hegemônicas no período $13,14,19,20$.

Em relação aos veículos de circulação das ideias e às instâncias do processo de organização, mobilização e luta dos profissionais do campo no período 1930-1963, vale destacar que: (1) os Arquivos brasileiros de nutrição, uma das inúmerasidealizações de osuéde Castro, constituíramse no mais importante veículo de difusão do saber científico em Alimentação eN utrição produzido no país no período ora investigado ${ }^{7,9}$; (2) 0 livro Alimentação brasileira à luz da geografia humana, publicado por JosuédeCastro em 1937, foi o principal veículo de circulação da tese "do mal de fome e não de raça" 13,14,19,20; (3) o clássico Geografia da fome, publicado por Josué Castro em 1946, por sua vez, traçava pela primeira vez 0 mapa da fome e das carências alimentares e nutricionais do país, além de ser um dos principais difusores das teorias vigentes sobre o nacional desenvolvimentismo ${ }^{13,14,19,20 ;}$; (4) a SociedadeBrasileira de Nutrição (SBN), entidade de caráter técnico-científico e cultural, fundada em 1940 , passaria a defender os interesses dos profissionais do campo, particularmente dos médicosnutrólogos ${ }^{7,9,10}$; e (5) a Associação Brasileira de Nutricionistas (ABN), fundada em 31 de agosto de 1949, foi a entidade brasileira criada com 0 intuito derepresentar edefender osinteresses dos nutricionistas/dietistas ${ }^{10}$.

\section{A consolidação do campo (1964 a 1984)}

No período de 1964 a1984, em face das contradiçõesinerentes ao contexto deditaduramilitar, identificam-se a emergência das pesquisas nutricionais de base populacional com amostras repre sentativas de todas as regiões geográficas do país; as tentativas de incorporação de técnicas de planejamento nutricional ao planejamento econômico, conduzidas sob a direção do então Instituto N acional de Alimentação e Nutrição (I nan); a expansão do número de cursos de graduação de nutrição; a criação de cursos superiores para formação de novas categorias profissionais do campo (tecnólogos e engenheiros de alimentos) e 0 início dos primeiros cursos de pós-graduação em Alimentação eN utrição lato sensu (especialização) e stricto sensu (mestrado e doutorado) ${ }^{10-12}$.

No cenário internacional, no início da década de 1970 assistiu-se a um contexto de crise do capitalismo, em que a questão da fome mundial ressurgiu com maior intensidadea partir da controvertida crise mundial dealimentos. No Brasil, a degradação das condições de vida das massas trabalhadoras excluídas do processo de crescimento econômico foi atestada por vários estudos, particularmente pelo Estudo Nacional de Despesas Familiares (Endef), realizado em 1974/ 1975. A partir dessa conjuntura, organismos internacionais como a OM S, a FAO eo Unicef passaram a defender a necessidade da incorporação do planejamento nutricional ao planejamento econômico dos países do Terceiro Mundo, em particular dos países latino-americanos. No Brasil, surgem o I e o II Planos $\mathrm{N}$ acionais de Desenvolvimento (PNDs), incorporando ao planejamento econômico instrumentos de políticas sociais. N essecontexto, por meio da Lei $n^{\circ} 5.829$, de 30/11/72, foi criado o Instituto Nacional de Alimentação eN utrição (Inan). Sob a tutela do Inan, em fevereiro de 1976 foi instituído o II Programa Nacional deAlimentação eN utrição (II Pronan), quedeveria ter duração coincidentecom o II PND (1975-1979), mas teve prorrogada sua execução até 1984,10-12.

Ressalta-se que a partir do II Pronan a questão nutricional voltou a assumir um lugar de destaquena agenda pública, constituindo um dos principais instrumentos da política social conduzida pelos governos militares. Com a instituição do II Pronan, outro médico pernambucano voltava a ocupar o posto mais elevado da principal agência pública do campo da Nutrição no Brasil: dr. Bertoldo Kruse Grande de Arruda, 0 qual presidiu o Inan no período de 1974 a 1984. Antes disso, outras agências da política de alimentação enutrição, tais como o SAPS ea CNA, haviam sido presididas por Josué deC astro. Destaca-se queem 1964, com a instalação da ditadura militar, Josué de Castro foi exilado do país, falecendo em Parisno ano de 1973. Além disso, é preciso reconhecer que foi a partir do desenvolvimento dos programas do II Pronan que ocorreu o processo de institucionalização de ações de Alimentação e Nutrição no interior da rede pública de serviços de saúde, educação, assistência social etc. em todo o território nacional ${ }^{7,10-12}$.

Em relação aos cientistas protagonistas do processo de consolidação do campo no período de 1964 a 1984, observou-se que, embora ainda 
concentrados no centro-sul do país, houve uma disseminação para outras regiões do Brasil. Em Pernambuco destaca-se o grupo de cientistas liderados por N elson Chaves, entre os quais apontamos: Bertoldo Kruse Grande de Arruda, Emília Aureliano de Alencar Monteiro, Heloísa de Andrade Lima Coêlho, M alaquias Batista Filho, $M$ aria Anunciada Ferraz de Lucena e M arly Cordeiro Baez ${ }^{9,11,13,15,21}$. Em São Paulo, no campo específico de AN SC, destaca-se o grupo de cientistas liderados por Yaro Ribeiro Gandra (Carlos Augusto Monteiro, Ignez Salas M artins, Sophia Cornbluth Szcarfarc, entre outros) e as nutricionistas do Instituto de Saúde, Bárbara Regina Lerner, Doris Lúcia M arlini Lei, M aria Lúcia Rosa Stefanini e Sandra Pinheiro Chaves. Ainda em São Paulo, destaca-se a importante contribuição de cientistas vinculados a outros campos que fazem interfaces com ANSC, tais como AIberto Carvalho da Silva, Franco M aria Lajolo, JoséEduardo Dutra deO liveira, Júlio Sérgio M archini, Hélio Vannuchi e Rebeca Carlota de Angelis. No Rio de Janeiro, destacam-se as atuações das nutricionistas Enilda Lins da Cruz Gouveia, Emília de Jesus Ferreiro, Lúcia Ypiranga Dantas Rodrigues, Maria Auxiliadora Santa Cruz Coe Iho, N euza Therezinha Rezende CavalcanteeSônia M oreira Alves, entre outras $5^{7,9,11,13,15}$.

Em relação às concepções científicas ou paradigmas que nortearam a atuação dos cientistas do campo no período de 1964 a 1984, conforme apontaram estudos anteriores, predominaram as abordagens multicausais da determinação do processo fome/desnutrição, baseadas em concepções de mundo positivistas e funcionalistas. NoS últimos anos da década de 1970 e nos primeiros anos da de 1980, as concepções estruturalistas da determinação do processo fome/desnutrição passaram a ser enfatizadas. Assim, as investigações científicas em Alimentação e N utrição passaram a identificar elementos estruturais como a distribuição da renda (a baixa renda), a distribuição da terra e outros elementos da forma de organização social como os principais determinantes do processo fome/desnutrição $0^{7,10-12,21,22}$.

Em relação aos veículos decirculação das ideias e às instâncias do processo de organização, mobilização e luta dos profissionais do campo no período de 1964 a 1984, vale destacar que:

(1) 0 clássico N utrição básica eaplicada, publicado em 1978 por Nelson Chaves, tornou-se uma das principais referências bibliográficas do processo didático-pedagógico de formação do nutricionista e outros profissionais do campo. Além disso, se constituiu num dos principais veículos de circulação das ideias do grupo de cientistas liderados por N elson Chaves, contribuindo para definir quais os problemas e os métodos legítimos do campo no Brasil. Ou seja, a visão ecológico-humanista acerca dos métodos de diagnóstico, prevenção e tratamento do processo fome/desnutrição, contida em Nutrição básica e aplicada, constituir-se-ia no paradigma dominante da Alimentação e Nutrição no Brasil, seja como ciência, seja como profissão e política pública ${ }^{11,13,21}$.

(2) Os Arquivos brasileiros de nutrição param de circular em 1968, provocando, por mais de uma década, um importante vazio no processo de circulação das ideias dos cientistas do campo a partir de então?.

(3) Em 08/04/1967 ocorreu a criação da Sociedade Brasileira de Ciência e Tecnologia de Alimentos (SBCTA), sediada no Instituto de Tecnologia de Alimentos (ITAL), em Campinas, São Paulo, com objetivos técnico-científicos esociais voltados aos profissionais do campo da Ciência e Tecnologia de Alimentos. Em janeiro de 1981, foi lançada a primeira edição da revista Ciência e Tecnologia de Alimentos, da SBCTA, a qual passou a se constituir no principal periódico de circulação das ideias em Ciência eTecnologia deAlimentos no Brasil (www.sbcta.org.br).

(4) Em 1972, a ABN transforma-se em Federação Brasileira das Associações de Nutricionistas (Febran), passando a congregar as associações técnico-científicas e culturais existentes em distintos estados brasileiros. Em 1980, foi lançado o primeiro número da revista Alimentação \& N utrição, periódico editorado pela Febran, o qual passou a ser importante veículo de circulação de ideias no campo da Alimentação e Nutrição ${ }^{10}$.

(5) Em 1973, no Rio deJaneiro, por iniciativa dos médicos J osé Evangelista e Clara Sambaquy Evangelista, foi criada a Associação Brasileira de Nutrologia(Abran), entidadetécnico-científicae social visando congregar os interesses dos médicos nutrólogos brasileiros.

De acordo com informações fornecidas pela Abran, em 1978 a Nutrologia foi reconhecida como especialidade médica pelo Consel ho Federal de M edicina (CFM ), pela Associação M édica Brasileira (AM B) e pelo Conselho Nacional de Residência M édica(CNRM) (www.abran.org.br). 
Os novos paradigmas

ea ressignificação do campo (1985 a 2010)

$N$ as duas últimas décadas, observou-se que ao perfil epidemiológico nutricional que caracterizou a sociedade brasileira no período 1930-1980 constituído pelas doenças relacionadas às carências nutricionais (desnutrição proteico-calórica, hipovitaminose A, pelagra, anemia ferropriva, bócio etc.), sobrepuseram-se as doenças relacionadas ao excesso nutricional (obesidade, diabe tes, dislipidemias, hipertensão, certos tipos de câncer etc.). Esteprocesso reconhecido como transição nutricional brasileira tem imprimido a ne cessidade de construção de novos enfoques explicativos eintervencionistas ${ }^{22-25}$.

Em relação à intervenção social do Estado brasileiro nesse terceiro corte analisado (19852010), correspondente aos governos J osé Sarney, Fernando Collor/Itamar Franco, Fernando Henrique Cardoso e Luís I nácio Lula da Silva, observou-se que a política pública de alimentação e nutrição transitou do planejamento autoritário ao participativo; da centralização à descentralização administrativa; da universal ização à focalização de benefícios; do controle estatal ao social; dos programas de distribuição de alimentos em espécie aos de tíquetes e aos de transferência de renda em dinheiro; do financiamento público às parcerias entre público e privado, entresociedade civil eEstado, entreinstituições governamentaise não governamentais; das ações emergenciais ou assistenciais às mediatas ou estruturais; das ações compensatórias às de emancipação ${ }^{11}$.

Em relação aos cientistas que protagonizam o processo de ressignificação do campo no período de 1985 a 2010, podemos dizer que ocorreu uma ampliação ediversificação do elenco de protagonistas, além de demarcação mais nítida de subcampos de atuação no interior da ANSC. Assim, podemos identificar pelo menos três subcampos de atuação compondo o campo da ANSC: Epidemiologia Nutricional; Política, Planejamento e Gestão em Alimentação e N utrição; e Ciências Sociais e H umanas em Alimentação e Nutrição ${ }^{25}$. No subcampo Epidemiologia Nutricional, que engloba todo o amplo conjunto das doenças nutricionais relacionadas à carência e/ ou excesso de nutrientes, destacamos a atuação de Ana M arlucia Oliveira Assis, Carlos Augusto M onteiro, César Gomes Victora, Denise Petrucci Gigante, Gilberto Kac, Leonor Maria Pacheco Santos, Luiz Antonio dos Anjos, M alaquias Batista Filho, Pedro Israel Cabral de Lira, Rosely Sichieri, entre outros. No subcampo de Política,
Planejamento eGestão em Alimentação e Nutrição, destacamos Ana Beatriz Vasconcellos, DeniseCoitinho, Flávio Luiz Schieck Valente, Francisco de Assis Guedes de Vasconcelos, Inês Rugani Ribeiro de Castro, Luciene Burlandy Campos de Alcântara, Renato Sergio Jamil M aluf, Sandra M aria Chaves dos Santos, entre outros. No subcampo das Ciências Sociais e Humanas em Alimentação e Nutrição, destacamos Ana M aria Canesqui, Dulce Lopes Barboza Ribas, Eronides da Silva Lima, Ligia Amparo da Silva Santos, M aria do Carmo Soares de Freitas, M aria Lúcia M agal hães Bosi, Rosa Wanda Diez Garcia, Rosana M agalhães, Shirley Donizete Prado, entre outros ${ }^{25}$. Vale destacar que a atuação nestes campos não é um processo excludente, observandose que muitos dos protagonistas do campo transitam livremente pelos três subcampos.

Em relação às concepções científicas ou paradigmas que nortearam a atuação dos cientistas do campo no período de 1985 a 2010, observouse a partir dos últimos anos 1980 a influência da concepção da determinação histórico-social do processo saúde/doença nas investigações epidemiológicas nutricionais. Neste sentido, alguns estudos empíricos divulgados naquel es anos utilizaram uma operacionalização do conceito de classe social, demonstrando ser esta categoria extremamente útil na identificação e explicação das desigualdades verificadas no processo saúde-doençą ${ }^{26,27}$. Ao longo da década de 1990, entretanto, a partir da crise do chamado socialismo real, caracterizada pela queda do muro de Berlim, pela perestroika soviética e pelo retorno à sociedade de mercado ocorrido nos países socialistas, verificou-se certa retração da concepção histórico-social em detrimento do recrudescimento das doutrinas neoliberais ${ }^{11,22}$. Assim, dentro da nova ordem econômica mundial que se estabelecia, também instalou-se o quese chamou de crise paradigmática, de busca de novos paradigmas $^{28,29}$. No campo da Alimentação e Nutrição brasileira, também têm sido verificadas tentativas de busca de novos paradigmas que possibilitem a apreensão da complexa realidade alimentar e nutricional que vem se configurando a partir de então. N este sentido, têm sido identificados distintos pontos de vista sobre este paradoxo nutricional brasileiro, tais como aqueles centrados no "enfoque da transição epidemiológica nutricional"23,24,30, na "análise ecológica da obesidadee outras doenças não transmissíveis" ${ }^{31}$, na "concepção holística da N utrição" 32, na "abordagem fenomenológica da fome" ${ }^{33}$, na "visão sobre 0 direito humano à alimentação e nutri- 
ção" 34 e mesmo na "teoria da determinação social da doença" ${ }^{35}$.

Em relação aos veículos de circulação das ideias e às instâncias do processo de organização, mobilização e luta dos profissionais do campo no período de 1985 a 2010, vale destacar:

(1) A ampliação do número de periódicos brasileiros veiculando as ideias do campo: Revista deN utrição (início decirculação em janeiro de 1988), Revista de Saúde Pública (desde junho de 1967), Cadernos de Saúde Pública (início em janeiro/março de 1985), Ciência \& Saúde Coletiva (início em 1996), Revista Brasileira deEpidemiologia (início em 1998), Revista Brasileira de Saúde M aterno-Infantil (início em 2001), Jornal de Pediatria (desde 1934), N utrire(início em 1990), entre outros.

(2) A ampliação das publicações em formato de livros, entre eles: Fome e desnutrição: determinantes sociais, organizado por Flávio Luiz Schieck Valente, com primeira edição em 1986; Epidemiologia da desigualdade: um estudo longitudinal de 6.000 crianças brasileiras, organizado por César G. Victora, Fernando C. Barros e J. Patrick Vaughan, com primeira edição em 1988; Saúde enutrição das crianças de São Paulo: diagnóstico, contrastes sociais e tendências, de Carlos Augusto M onteiro, com primeira edição em 1988; A face oculta da Nutrição, de M aria Lúcia Magalhães Bosi, com primeira edição em 1988; Repensando a desnutrição como questão social, de Paulete Goldenberg, com primeira edição em 1988; Velhos e novos males da Saúde no Brasil: a evolução do país e de suas doenças, organizado por Carlos Augusto M onteiro, com primeira edição em 1995; Nutrição, Saúde \& comunidade, organizado por Enilda Lins da Cruz Gouveia, com primeira edição em 1992; Avaliação nutricional de coletividades, de Francisco de Assis Guedes de Vasconcelos, com primeira edição em 1993; Direito humano à alimentação: desafios e conquistas, organizado por Flávio Luiz Schieck Valente, com primeira edição em 2002; Agonia da fome, de Maria do Carmo Soares de Freitas, com primeira edição em 2003; Antropologia e nutrição: um diálogo possível, organizado por Ana M aria Canesqui e Rosa Wanda Diez Garcia, com primeira edição em 2005; Epidemiologia Nutricional, organizado por Gilberto Kac, Rosely Sichieri eD enise P. Gigante, com primeira edição em 2007; e0 corpo, o comer ea comida: um estudo sobre as práticas corporais e alimentares no mundo contemporâneo, de Ligia Amparo da Silva Santos, com primeira edição em 2008.

(3) A ampliação do número de entidades técnico-científicas e culturais atuando no campo, destacando-se: (a) a Associação Brasileira dePósGraduação em Saúde Coletiva (Abrasco), criada em 27 de setembro de 1979 (www.abrasco. org.br); (b) a Associação Brasileira de N utrição (Asbran) (continuidade da Associação Brasileira de $N$ utricionistas, fundada em 31 de agosto de 1949) (www.asbran.org.br); (c) a Sociedade Brasileira de Alimentação e Nutrição (SBAN), fundada em 31 dejulho de 1985 (www.sban.com.br); e (d) a Associação Brasileira para o Estudo da O besidade (Abeso), criada em 14 de agosto de 1986 (www.abeso.org.br).

\section{Consideraçõesfinais}

Resta-nos sinalizar que o campo da ANSC assumiu a sua atual configuração e ressignificação a partir de meados da década de 1970, no interior do chamado movimento sanitário brasileiro, cujo principal ideário foi a realização da reforma sanitária e a construção do Sistema Ú nico de Saúde (SUS) ${ }^{36}$.

0 engajamento de profissionais do campo da ANSC nas atividades promovidas pela Abrasco constituiu um relevante fator determinante da atual configuração do campo. Entre tais atividades, destacam-se a participação na 8 a Conferência Nacional deSaúde (8a CNS), realizada em Brasília, no período de 17 a 21 de março de 1986; no I Congresso Brasileiro de Saúde Coletiva, realizado no Rio de Janeiro, de 22 a 26 de setembro de 1986; enal Conferência N acional deAlimentação e Nutrição (I CNAN), um dos desdobramentos da 8a CNS, realizada em Brasília, entre 26 e 28 de novembro de 1986, quando se assumiu pela primeira vez a premissa de que 0 acesso à alimentação adequada nos diferentes estágios biológicos é um direito fundamental detodas as pessoas euma obrigação prioritária do Estado ${ }^{34,37}$.

Ao longo da tortuosa trajetória histórica entreal CN AN (novembro de 1986) ea promulgação do Projeto deEmenda Constitucional (PEC) no 47 , de 4 de fevereiro de 2010, que incluiu no artigo 60 da Constituição de 1988 a alimentação como um dos direitos sociais de todo brasilei$\mathrm{ro}^{38}$, destaca-se a realização de uma ampla relação de eventos, fatos históricos, ações e serviços relacionados direta ou indiretamente ao processo de construção do SU Se conquista do direito à saúde e à alimentação, os quais tiveram a relevante participação dos profissionais do campo da ANSC. Como ilustração, destacamos: (1) a criação do Sistema Nacional de Vigilância Alimentar e Nutricional (Sisvan), por meio da Portaria do MS no 1.156, de agosto de 199011; (2) a 
constituição da Ação da Cidadania contra a Fome, a M iséria e pela Vida, liderada por Herbert de Souza (Betinho) a partir de 199339; (3) as Conferências Nacionais de Segurança Alimentar realizadas a partir de 199434; (4) a emissão da Portaria no 710 do MS, de 10 de junho de 1999, que aprovou a Política Nacional de Alimentação e Nutrição (PNAN) 40; (5) o Programa Fome Zero, lançado em janeiro de $2003^{11}$; (6) o ConseIho Nacional deSegurança Alimentar eN utricional (Consea), criado a partir da M edida Provisória $\mathrm{n}$ 은 102, de 10 de janeiro de 2003 e regulamentado pelo Decreto no 4.582, de 30 de janeiro de 2003무 ( 7) a instituição do Sistema Nacional deSegurança Alimentar eN utricional (Sisan), a partir da Lei no 11.346, de 15 de setembro de 200641; e (8) a aprovação da Lei no 11.947, de 16 de julho de 2009, que entre outras providências amplia a cobertura do Programa Nacional de Alimentação Escolar (PNAE) para toda a rede pública e filantrópica de educação básica ${ }^{42}$.

Por fim, reafirma-se tanto a importância do caráter multidisciplinar como a premissa do relevanteeindiscutível papel dos profissionais do campo da AN SC na garantia do direito humano à alimentação saudável, um dos componentes do conjunto decondições eserviçosnecessáriosà prevenção de doenças, promoção, manuten ção e recuperação da saúdee, consequentemente, melhoria da qualidade de vida de todos os brasileiros.

\section{Colaboradores}

FAG Vasconcelos e M Batista Filho trabalharam de forma articulada e simultânea em todas as etapas do processo de construção do manuscrito: concepção teórica, levantamento e revisão da literatura, redação e revisão final do artigo. 


\section{Referências}

1. Bourdieu P. El campo científico. Revista de Estudios Sociales de la Ciencia 1994; 1(2):131-160.

2. Bourdieu P. 0 poder simbólico. $2^{\text {a }}$ ed. Rio de Janeiro: Bertrand Brasil; 1998.

3. Kuhn TS. A estrutura das revoluções científicas. $5^{\mathrm{a}} \mathrm{ed}$. São Paulo: Perspectiva; 1997.

4. Cannon $G$, Leitzmann $C$. The new nutrition science project. Public Health Nutr 2005; 8(6A):673-694.

5. MCM ichael AJ. Integrating nutrition with ecology: balancing the health of humans and biosphere. Public Health Nutr 2005; 8(6A):706-715.

6. Afman L, Müller M. Nutrigenomics: from molecular nutrition to prevention of disease. J Am Diet Assoc 2006; 106:569-576.

7. L'Abbate S. As Políticas de Alimentação e Nutrição no Brasil: I. Período de 1940 a 1964. Rev Nutr 1988; 1(2):87-138.

8. Ypiranga L. Delimitação do objeto de trabalho do nutricionista: subsídios para uma discussão. Saúde em Debate 1990; 29:62-69.

9. Vasconcelos FAG. Os Arquivos brasileiros de nutrição: uma revisão sobre produção científica em Nutrição no Brasil (1944 a 1968). Cad Saude Publica 1999; 15(2):303-316.

10. Vasconcelos FAG. 0 nutricionista no Brasil: uma análise histórica. Rev Nutr 2002; 15(2):127-138.

11. Vasconcelos FAG. Combate à fome no Brasil: uma análise histórica de Vargas a Lula. Rev Nutr 2005; 18(4):439-457.

12. Arruda BKG, Arruda IKG. Marcos referenciais da trajetória das políticas de alimentação e nutrição no Brasil. Rev Bras Saude M ater Infant 2007; 7(3):319326.

13. Vasconcelos FAG. Origem e conformação do campo da nutrição em Saúde Pública em Pernambuco: uma análise histórico-estrutural. Rev Nutr 2001; 14(Supl.):12-18.

14. Vasconcelos FAG. Fome, eugenia e constituição do campo da Nutrição em Saúde Pública em Pernambuco: uma análise de Gilberto Freyre, Josué de Castro e Nelson Chaves. Hist Ciên Saúde - M anguinhos 2001; 8(2):315-339.

15. Dutra-de-Oliveira JE, Marchini JS. Nutritional sciences in Brazil: the pioneer work of institutions and scientists. Nutr 2004; 20(2):174-176.

16. Aperibense PGGS, Barreira IA. A enfermeira Liese lotte Hoeschl Ornellas e o surgimento da profissão de nutricionista. Esc Anna Nery 2006; 10(3):560564.

17. Aperibense PGGS, Barreira IA. N exos entre Enfermagem, Nutrição e Serviço Social, profissões femininas pioneiras na área da Saúde. Rev Esc Enferm USP 2008; 42(3):474-482.

18. Governo do Estado de São Paulo. Centro Paula Souza. Da alimentação à nutrição: 70 anos de educação profissional (1939-2009). [acessado 2010 mar 10]. Disponível em: http://www.cpscetec.com.br/memorias/70_anos/historico.html

19. Lima ES. Gênese e constituição da educação alimentar: a instauração da norma. Hist Ciên Saúde - M anguinhos 1998; 5(1):57-84.

20. M agalhães R. Fome: uma (re) leitura de Josué de Castro. Rio de Janeiro: Editora Fiocruz; 1997.
21. Vasconcelos FAG. Um perfil de Nelson Chaves e da sua contribuição à Nutrição em Saúde Pública. Cad Saude Publica 2001; 17(6):1505-1518.

22. Vasconcelos FAG. Tendências históricas dos estudos dietéticos no Brasil. Hist Ciên Saúde - Manguinhos 2007; 14(1):197-219.

23. Batista Filho M, Rissin A. A transição nutricional no Brasil: tendências regionais e temporais. $\mathrm{Cad}$ Saude Publica 2003; 19 (Supl.1):S181-S191.

24. Batista Filho M, Souza AI, Miglioli TC, Santos MC. Anemia e obesidade: um paradoxo da transição nutricional brasileira. Cad Saude Publica 2008; 24 (Supl.2):s247-s257.

25. Canesqui AM. Pesquisas qualitativas em Nutrição e Alimentação. Rev Nutr 2009; 22(1):125-139.

26. M onteiro CA. Saúde e nutrição das crianças de São Paulo: diagnóstico, contrastes sociais e tendências. São Paulo: Hucitec/EdUSP; 1988.

27. Victora CG, Barros FC, Vaughan JP. Epidemiologia da desigualdade: um estudo longitudinal de 6.000 crianças brasileiras. 2a ed. São Paulo: Hucitec; 1989.

28. Souza Santos B. U m discurso sobre as ciências. $7^{\text {a }} \mathrm{ed}$. Porto: Afrontamento; 1995.

29. Singer P. Ética prática. São Paulo: Martins Fontes; 1994.

30. M onteiro CA, M ondini L, Souza ALM, Popkin BM. Da desnutrição para a obesidade: a transição nutricional no Brasil. In: Carlos Augusto M onteiro, organizador. Velhos e novos males da Saúde no Brasil: a evolução do país e de suas doenças. $2^{2}$ ed. São Paulo: H ucitec/N upens-USP; 2000. p. 247-255.

31. Sichieri R. Epidemiologia da obesidade. Rio de Janeiro: Eduerj; 1998.

32. Bosi MLM . A Nutrição na concepção científica moderna: em busca de um novo paradigma. Rev Nutr 1994; 7(1):32-47.

33. Freitas MCS. U ma abordagem fenomenológica da fome. Rev Nutr 2002; 15(1):53-69.

34. Valente FLS, organizador. Do direito humano à alimentação: desafios e conquistas. São Paulo: Cortez; 2002.

35. Escoda MSQ. Para a crítica da transição nutricional. Cien Saude Colet 2002; 7(2):219-226.

36. M inayo MCS. Os 20 anos do SUS e os avanços na vigilância e na proteção à saúde. Epidemiol Serv Saúde 2008; 17(4):245-246.

37. Brasil. Ministério da Saúde, Ministério da Previ-

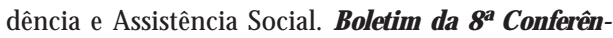
cia Nacional de Saúde, 9. Brasília; mar. 1987.

38. Brasil. Conselho Nacional de Segurança Alimentar e Nutricional (Consea). Agora é lei: alimentação é um direito; Consea celebra com parceiros. [online]. Brasília, 5 de fevereiro de 2010. [acessado 2010 fev 28]. Disponível em: https://www.planalto.gov.br/Consea/ exec/index.cfm

39. Brasil. Ação da Cidadania Contra a M iséria e pela Vida. Cartilha para ajudar a formar comitês. Brasília: Secretaria Nacional da Ação da Cidadania contra a M iséria e pela Vida; 1993. 
40. Brasil. M inistério da Saúde. Coordenação-Geral da Política de Alimentação e Nutrição. Política Nacional de Alimentação e Nutrição. 2a ed. rev. Brasília: Ministério da Saúde; 2003. [online]. [acessado 2010 fev 27]. Disponível em: http://nutricao.saude. gov.br/documentos/pnan.pdf

41. Brasil. Lei no 11.346, de 15 de setembro de 2006 . Cria o Sistema Nacional de Segurança Alimentar e Nutricional (SISAN) com vistas em assegurar o direito humano à alimentação adequada e dá outras providências [online]. [acessado 2010 fev 27]. Disponível em: https://www.planalto.gov.br/consea/ static/eventos/LOSAN $\% 20 \% 20$ Lei $\% 2011.346 \%$ 20de\%2015\%20de\%20setembro\%20de\%2020061.pdf

42. Brasil. Lei no 11.947, de 16 de junho de 2009. Dispõe sobre 0 atendimento da alimentação escolar e do Programa Dinheiro Direto na Escola aos alunos da educação básica [acessado 2010 fev 27]. Disponível em: http://www.fnde.gov.br/index.php/ae-legislacao

Artigo apresentado em 10/02/2010

Aprovado em 20/05/2010

Versão final apresentada em 12/07/2010 